\title{
Systematic Review and Meta-Analysis on the Efficacy of Chemotherapy with Transurethral Resection of Bladder Tumors as Definitive Therapy for Muscle Invasive Bladder Cancer
}

\author{
George W. Moran ${ }^{\mathrm{a}, *}$, Gen Li ${ }^{\mathrm{b}}$, Dennis J. Robins ${ }^{\mathrm{c}}$, Justin T. Matulay ${ }^{\mathrm{c}}$, James M. McKiernan ${ }^{\mathrm{c}}$ \\ and Christopher B. Anderson ${ }^{\mathrm{c}}$ \\ ${ }^{a}$ Columbia University College of Physicians and Surgeons, New York, NY, USA \\ ${ }^{\mathrm{b}}$ Department of Biostatistics, Mailman School of Public Health, Columbia University, New York, NY, USA \\ ${ }^{\mathrm{c}}$ Department of Urology, Columbia University Irving Medical Center, New York, NY, USA
}

\begin{abstract}
.
Background: Bladder-sparing treatment of muscle invasive bladder cancer (MIBC) with systemic chemotherapy plus transurethral resection of bladder tumors (TURBT) is increasingly seen in the literature - both in case series and subanalyses of patients who opt out of or are unfit for radical cystectomy (RC). Survival outcomes among these patients are often impressive, but these are typically small retrospective studies from single institutions and therefore of limited clinical value.

Objectives: Our aim is to summarize the literature regarding definitive treatment of MIBC with systemic chemotherapy plus TURBT and provide a meta-analysis of survival outcomes for patients who received this treatment.

Methods: A systematic literature search was performed consistent with the Prisma statement to identify publications reporting the outcomes of patients treated with TURBT and systemic chemotherapy as definitive treatment for locally confined MIBC. Identified studies were screened in a two-stage process: first by title and abstract; then by full-text reading. 18 publications (518 patients) were included in the qualitative systematic review and 10 publications (266 patients) were included in the meta-analysis. The primary objective was overall survival (OS).

Results: Overall survival ranged from $20 \%$ to $87.5 \%$ across studies at median follow-up ranging 4 to 120 months. 5-year survival rate for all patients included in the meta-analysis was estimated to be $72 \%$ [95\% CI: $64 \%, 82 \%$ ].

Conclusions: Definitive treatment with systemic chemotherapy plus TURBT can lead to favorable survival outcomes in select patients. Further study to improve patient selection for this method of treatment is needed.
\end{abstract}

Keywords: Bladder cancer, neoadjuvant therapy, chemotherapy

\section{INTRODUCTION}

*Correspondence to: George W. Moran, Department of Urology, Columbia University College of Physicians and Surgeons, Herbert Irving Pavilion, 161 Fort Washington, 11th Floor, New York, NY 10032, USA. Tel.: +1 212305 0114; E-mail: gwm2118@cumc.columbia.edu.
Bladder cancer ranks among the most common solid organ malignancies in the United States, with approximately 79,000 new diagnoses expected in 2017 and over 16,000 deaths [1]. While superficial, 
or non-muscle invasive, bladder cancer (NMIBC) comprises the majority of new cases, roughly onequarter of patients will present with muscle invasion at diagnosis [2]. The gold standard treatment of muscle invasive bladder cancer (MIBC) is neoadjuvant chemotherapy (NAC) plus radical cystectomy (RC) with pelvic lymphadenectomy [3, 4]. The largest randomized trial of patients receiving NAC plus RC for locally invasive MIBC reported 5- and 10-year overall survival (OS) of $49 \%$ and $36 \%$, respectively [5]. This treatment carries an extremely high morbidity, estimated to be as high as $66 \%$, and a perioperative mortality rate approaching 1 out of 20 (4.2\%) [6]. In addition, pathological examination of cystectomy specimens after RC with NAC will reveal that $30-40 \%$ of patients have no evidence of disease in their bladders at the time of cystectomy [7]. This leads one to question the merits of a "one-size-fits-all" approach to MIBC.

There is limited but growing interest among expert urologic oncologists that bladder-sparing approaches in the MIBC patient population may be safe and yield similar survival outcomes to NAC plus RC [8-14]. Support for trimodality therapy (TMT), which preserves the native bladder using systemic chemotherapy, complete transurethral resection of bladder tumors (TURBT), and external beam radiation therapy (XRT) is widespread, particularly among European urologists, but still introduces significant unwarranted morbidity for the pathologic complete responder. A less morbid approach would be to identify those patients who have achieved complete pathologic response (CR) before radical therapy and offer them bladder conservation with close follow-up. This method of systemic chemotherapy plus TURBT is increasingly seen in the literature in the form of case series and subanalyses of NAC studies with favorable survival outcomes reported as far out as 10 years following treatment [9-11, 15-24]. However, these studies are generally small, often from single institutions, and typically report retrospectively analyzed data with heterogeneous inclusion criteria and outcomes measures, thus limiting their ability to spur changes in the standard of care. Furthermore, these studies only report a fraction of all patients who have undergone this therapeutic regimen and can be found in the broader MIBC literature. Indeed, numerous smaller groups of patients who receive systemic chemotherapy and TURBT as definitive treatment, either incidentally or as an alternative to a different bladder-sparing regimen, are reported throughout the literature. The purpose of this review is to summarize the literature regarding the definitive treatment of MIBC with TURBT plus systemic chemotherapy and provide an analysis of survival outcomes for patients receiving this treatment.

\section{METHODS}

\section{Search strategy}

A systematic literature search was performed consistent with the Prisma statement [25] to identify publications reporting outcomes for patients treated with TURBT and systemic chemotherapy as definitive treatment for locally confined (i.e. no evidence of metastasis to lymph nodes or distant sites) muscle invasive transitional cell carcinoma of the bladder. Eligible studies included all English language manuscripts published in any year. Abstracts were only considered when they provided updates to patient cohorts already selected in full-length publications.

The Medline (via Pubmed) and Embase databases were initially searched for 18 different combinations of two or three of the following terms: muscle invasive bladder cancer, chemotherapy, transurethral resection, TURBT, bladder-sparing, conservative management, salvage therapy, MVAC, Gemcitabine, Cisplatin, and platinum therapy. Additionally, a manual search was performed of abstracts from the following meetings between 2013 and 2017: the Annual Meeting of the American Society of Clinical Oncology (ASCO), the Genitourinary Cancers Symposium, the Annual Meeting of the American Urological Association (AUA), the Annual Meeting of the Society of Urologic Oncology (SUO), the Annual Congress of the European Society for Medical Oncology (ESMO), and the Annual Congress of the European Association of Urology (EAU). Any updates to the outcomes reported in selected publications were incorporated into this review and analysis. All searches were conducted in March and April of 2017.

In searching the Pubmed database, search terms were separated by a "+." For the Embase search, each term was enclosed in "" and searched in "all fields" as separate search lines connected by "and."

\section{Study selection}

Search returns were evaluated in two stages. First, returns were screened by title and abstract, and publications were excluded for the following reasons (Fig. 1): repeat publications from different search 
terms or databases, non-original articles (reviews and systematic reviews with or without meta-analyses, consensus statements, editorial comments, and letters), case reports, meeting abstracts, non-English articles, publications reporting on all or part of the same cohort as that in another paper with a longer follow-up or later publication date, and publications explicitly unrelated to bladder-preservation for MIBC without radiotherapy. Second, full-text reading of all selected publications was performed to remove any publication without survival data reported specifically for patients with MIBC receiving an initial definitive therapy of TURBT plus systemic chemotherapy for locally confined MIBC.

Patients were excluded from this review if radical or partial cystectomy was planned immediately following post-systemic chemotherapy re-staging. However, patients who received salvage cystectomy, defined as a cystectomy planned in response to progression of disease detected after being approved for bladder sparing in light of initial post-systemic chemotherapy re-staging, were included. Any patient receiving radiotherapy at any point in his or her treatment was excluded. Those who received intravesical therapy before, following, or simultaneously with systemic therapy were included, though patients who received intravesical chemotherapy as their only chemotherapy were not. Any patient who received systemic chemotherapy only as a salvage treatment for disease progression was excluded. Finally, appropriate patients had to be excluded if the publication in which they were described did not report or permit extrapolation of numeric survival outcomes specific to patients and treatment meeting the criteria described above. When a portion of patients in a group were noted to have variant histology (adenocarcinoma, squamous cell carcinoma), $[19,26]$ the group was included in the review and tables with notation indicating this, but excluded from the meta-analysis. If the entire cohort had non-transitional cell histology, however, the publication was excluded.

\section{Data extraction/data items}

Where present, the following variables were extracted: number of patients, length of follow-up, clinical stage before systemic chemotherapy, clinical stage after systemic chemotherapy and TURBT, reason for which RC was not performed, type of systemic chemotherapy given, type of intravesical chemotherapy given, number of systemic chemotherapy cycles completed in initial treatment, follow-up strategy,
OS, DSS, percent of patients who recurred, percent of patients who progressed to metastatic disease, PFS, cystectomy-free survival, and other tumor characteristics (grade, size, multifocality). The first author of the one abstract included [24] was contacted directly to ascertain the end of that study's treatment period. Where indicated in Table 4, the assumption was made that the median follow-up for the entire study applied to the patients selected for our analysis as well.

\section{Outcome measures}

The primary objective of this study was OS. Two studies published only disease specific survival (DSS) and did not permit extrapolation of OS, [9, 27] so these patients were included in our review and tables but excluded from the meta-analysis. Due to inconsistency of reported outcomes between studies, recurrence, DSS, progression-free survival (PFS), and cystectomy-free survival could not be compared.

\section{Statistical analysis}

The software package "metafor" (Version 1.9-9) in $\mathrm{R}$ (Version 3.2.1) was used to conduct the metaanalysis on hazard rates. Hazard rates were induced from reported survival rates, assuming the survival rates follow an exponential distribution in each study. Hazard rates, signifying rate of death, were used to indicate the chance of survival for selected patients from each study. Fixed and random effects models were fitted to the data. Heterogeneity tests were conducted to select between the two types of models. If $p$-value $<0.05$, a random effects model was used, and vice versa. We provided a forest plot to show effect sizes and confidence intervals for individual studies as well as the meta-analysis. $\mathrm{I}^{2}$ analysis was used to represent heterogeneity among studies. We additionally provided a funnel plot in Supplementary Figure 1 to represent the likelihood of publication bias in each publication.

\section{RESULTS}

\section{Study selection}

The selection process for included studies is described in a CONSORT diagram provided in Fig. 1. The initial search of both databases yielded 7,366 returns, of which 2,499 were unique. Initial assessment resulted in exclusion of 2,438 publications. Of the 61 texts that underwent full-text review for rele- 


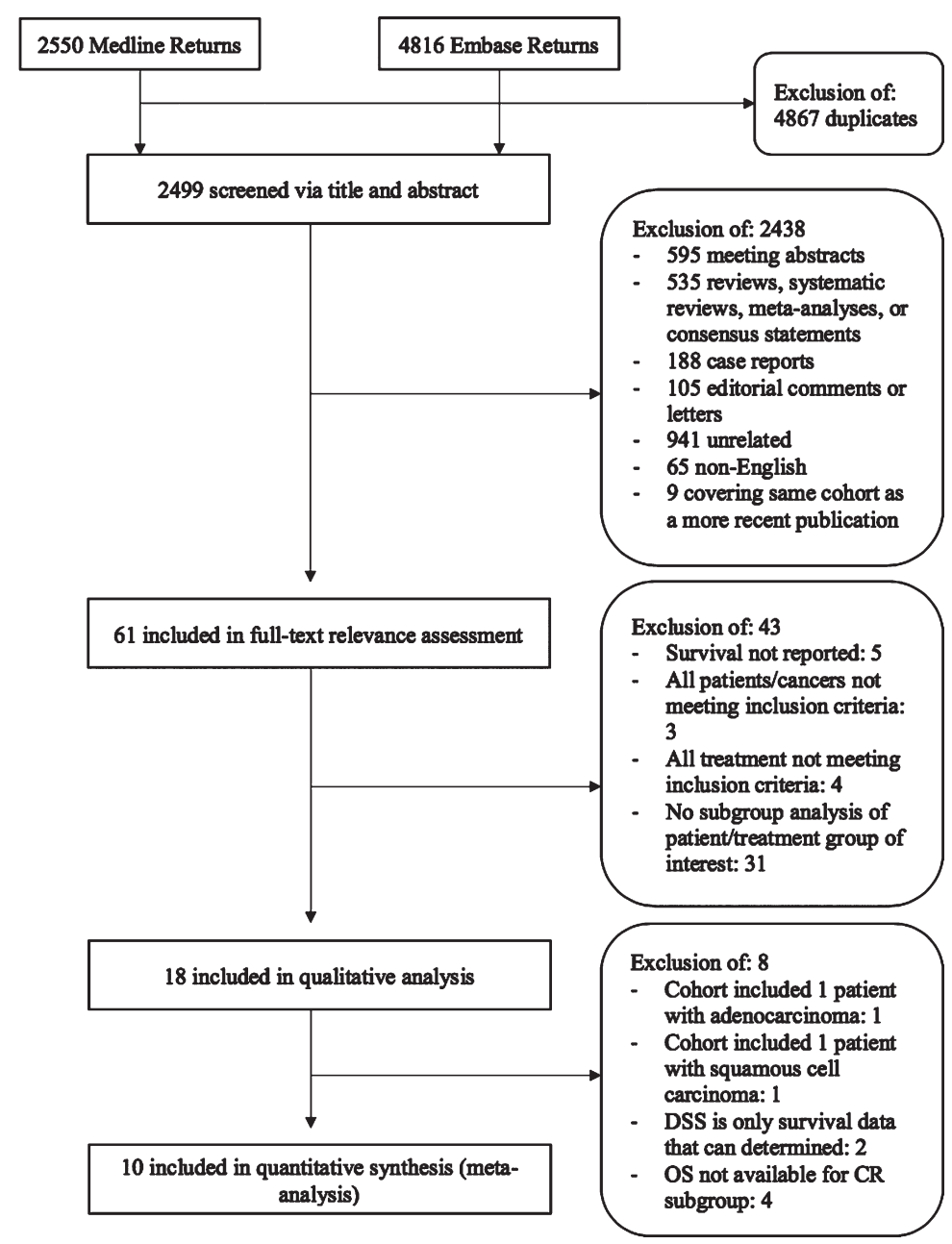

Fig. 1. CONSORT diagram of studies included in systematic review and meta-analysis.

vance, 18 were found to include patients who met the criteria. This amounted to 518 patients in total. From the 18 publications finally included, 2 were withheld from the meta-analysis because the cohorts each included one patient with variant histology, 2 others were removed because DSS was the only survival data that could be determined. Because this metaanalysis aims to ascertain the likelihood of survival for patients who elect bladder sparing following clinical complete remission, 4 studies from which the OS of the clinical complete responder subgroup could not be determined were also eliminated. Thus, 10 studies (266 patients) were included in the meta-analysis.

\section{Study characteristics}

As indicated in Table 1, patients included in the selected studies were treated between 1982 and 2014.
Patients included ranged in age from 30 to 92 years, with median ages by study ranging between 55.5 and 81. The largest systemic chemotherapy + TURBT cohort comprised 75 patients, while the smallest had 4. All included patients had locally-invasive bladder cancer on pre-treatment staging (T2NOM0, T2-3N0M0, or T2-T4NOM0).

All patients underwent treatment with transurethral resection of the bladder as well as a platinumbased regimen of systemic chemotherapy. As indicated in Table 3, the possible exception to this concerns the two complete responders from the study by Damyanov et al. [27] which is included in our discussion but excluded from the meta-analysis, and for which the method of initial staging was not specified. Four studies used MVAC (Methotrexate, Vinblastine, Doxorubicin, and Cisplatin) only, three used CMV (Cisplatin, Methotrexate, and Vinblastine) 
Table 1

Study and patient characteristics

\begin{tabular}{|c|c|c|c|c|c|c|}
\hline Authors & Study Period & $\mathrm{N}$ & $\begin{array}{c}\text { Median Age of } \\
\text { Patients Included in } \\
\text { This Review/Entire } \\
\text { Source Publication } \\
\text { (range) [yrs.] }\end{array}$ & $\begin{array}{l}\text { Functional Performance/Comorbitidy Score for } \\
\text { Entire Study }\end{array}$ & $\begin{array}{l}\text { Clinical Stage before } \\
\text { Systemic } \\
\text { Chemotherapy }\end{array}$ & $\begin{array}{l}\text { Clinical Stage after Systemic } \\
\text { Chemotherapy }\end{array}$ \\
\hline Bamias et al. [26] & 2001-2012 & 25 & $79(53-87)$ & $\begin{array}{l}\text { ECOG Performance Status } \leq 2 ; \text { Median } \\
\text { Charlson Comorbidity Score } 5 \text { (range 3-8)* }\end{array}$ & $\mathrm{T} 2-\mathrm{T} 4 \mathrm{aN} 0 \mathrm{M} 0^{\wedge}$ & - \\
\hline Damyanov et al. [27] & 1996-2000 & 14 & $55.7(40-68)$ & ECOG Performance Status $\leq 2^{*}$ & T2-T4NXM0 & $\begin{array}{l}\text { CR (T0) }(n=2) ; \mathrm{PR}(>/=50 \% \text { decrease } \\
\text { in tumor mass, no new lesions) }(n=8), \\
\text { NR (no change or progression) }(n=4)\end{array}$ \\
\hline de la Rosa et al. [15] (MA) & 1987-1990 & 18 & $60(44-75)$ & ECOG Performance Status $\leq 2^{*}$ & T2-T4NXM0 & TONOM0 \\
\hline $\begin{array}{l}\text { deVere White et al. [34] } \\
\text { (MA) }\end{array}$ & 2003-2007 & 24 & $69(49-83)$ & $\begin{array}{l}\text { ECOG Performance Status } \leq 2 \text { required for } \\
\text { inclusion; all patients were either } 0,1 \text {, or } \\
\text { missing* }\end{array}$ & T2-T4aN0M0 & TONOM0 \\
\hline Given et al. [33] (MA) & 1986-1990 & 7 & $65(45-83)$ & - & T2-T4N0M0 & TONOM0 \\
\hline Herr, 2008 [10] (MA) & 1995-2001 & 63 & $62(32-79)$ & - & T2-T4N0M0 & TONOM0 \\
\hline Herr et al. [16] (MA) & 1985-1989 & 28 & $64(30-79)$ & $\begin{array}{l}\text { Median Karnofsky Performance Status: } 90 \% \\
\quad(\text { range } 40-100 \%)^{*(1)}\end{array}$ & T2-3N0M0 & T0N0M0 \\
\hline Kondás et al. [17] & 1990-? & 33 & mean: 69 (51-87) & - & T2-T3N0M0 & - \\
\hline $\begin{array}{l}\text { Kuroiwa et al. [18] } \\
\text { (MA) }\end{array}$ & Published 1995 & 6 & $76(64-88) / 72(35-88)$ & ECOG Performance Status $\leq 2^{*}$ & T2-T3bN0M0 & $\begin{array}{l}\text { T0N0M0 }(n=1), \text { TaN0M0 }(n=2) \\
\quad \text { T1N0M0 }(n=3)\end{array}$ \\
\hline Lekili et al. [19] & $1991-1993$ & 30 & $55.5(44-67)$ & $\begin{array}{l}\text { Median Karnofsky Performance Status: } 80 \% \\
\quad(60-90 \%)^{*}\end{array}$ & $\mathrm{~T} 2-\mathrm{T} 3^{\wedge \wedge}$ & $\begin{array}{l}\text { T0N0M0 }(n=8), \text { T1N0M0 }(n=4), \\
\text { T2N0M0 }(n=8), \text { T4N+M+ }(n=10)\end{array}$ \\
\hline Li et al. [20] & 2007-2014 & 36 & mean: $78.6+/-5.3$ & - & T2-T3 & - \\
\hline Lodde et al. [31] & 2000-2004 & 4 & $83.5(77-89) / 81(68-92)$ & Median ASA Score: 3 (range 2-3) & T2NOM0 & - \\
\hline Matulay et al. [24] (MA) & 1988-2014 & 36 & mean: $67.2+/-9.6$ & - & T2-T3N0M0 & TONOM0 \\
\hline Shimizu et al. [21] & 1991-2001 & 8 & $71(60-78) / 71.5(60-86)$ & - & T2aNOM0 & - \\
\hline Solsona et al. [9] & 1989-2005 & 75 & 62 & - & T2-T4N0M0 & $\begin{array}{l}\text { T0 }(n=40) \text {, Ta1-Tis }(n=12), \mathrm{T}>/=2 \text { or } \\
\quad \mathrm{N}+\text { or } \mathrm{M}+(n=23)\end{array}$ \\
\hline $\begin{array}{l}\text { Sternberg et al. [11] } \\
\text { (MA) }\end{array}$ & 1987-2001 & 52 & $65(30-82)$ & $\begin{array}{l}\text { Median Karnofsky Performance Status: } 100 \% \\
\text { (range } 60-100 \%)^{*}\end{array}$ & T2-T4N0M0 & $\begin{array}{l}\text { T0 }(n=37), \text { TaN0M0 }(n=5), \text { Tis }(n=2), \\
\text { T1 }(n=4), \text { T2N?M+ }(n=1) ; 3 \text { patients } \\
\text { refused re-TURBT }\end{array}$ \\
\hline $\begin{array}{l}\text { Uygur et al. [22] } \\
\text { (MA) }\end{array}$ & $1990-1995$ & 16 & $62(46-75)$ & $\begin{array}{l}\text { Median Karnofsky Performance Status: } 90 \% \\
\text { (range } 80-100 \%)^{*}\end{array}$ & T2-T3aN0M0 & $\begin{array}{l}\text { T0N0M0 }(n=13), \text { Ta+TisN0M0 }(n=1), \\
\text { T2-T3aN0M0 }(n=2)\end{array}$ \\
\hline $\begin{array}{l}\text { Villavicencio et al. [23] } \\
\text { (MA) }\end{array}$ & 1982-1998 & 43 & mean: $61(45-75)$ & ECOG Performance Status $\leq 2^{*}$ & T2-T3aNOM0 & $\begin{array}{l}\text { T0NOM0 }(n=39) ; \text { T1- or TisN0M0 } \\
\quad(n=4)\end{array}$ \\
\hline
\end{tabular}

(MA) included in meta-analysis. ${ }^{*}$ value applies to entire study group, not just those meeting clinical criteria for inclusion in this analysis. ${ }^{\wedge} 1$ patient in entire publication with adenocarcinoma.

$\wedge \wedge 1$ patient in entire publication with squamous cell carcinoma. ${ }^{(1)}$ Schultz PK, Herr HW, Zhang ZF, et al. Neoadjuvant chemotherapy for invasive bladder cancer: prognostic factors for survival of patients treated with M-VAC with 5-year follow-up. J Clin Oncol 1994;12: 1394-401. 
Table 2

Reason for bladder sparing

\begin{tabular}{ll}
\hline Authors & Reason for Bladder Sparing \\
\hline Bamias et al. [26] & Unfit for RC \\
Damyanov et al. [27] & Refused RC \\
de la Rosa et al. [15] (MA) & - \\
deVere White et al. [34] (MA) & Refused RC \\
Given et al. [33] (MA) & Refused RC or Unfit for RC \\
Herr [10] (MA) & Refused RC \\
Herr et al. [16] (MA) & Refused RC \\
Kondás et al. [17] & RC "could not be performed for various reasons" \\
Kuroiwa et al. [18] (MA) & Refused RC or Unfit for RC \\
Lekili et al. [19] & - \\
Li et al. [20] & Refused RC or Unfit for RC \\
Lodde et al. [31] & Refused RC or Unfit for RC \\
Matulay et al. [24] (MA) & Refused RC \\
Shimizu et al. [21] & - \\
Solsona et al. [9] & Refused RC \\
Sternberg et al. [11] (MA) & Selected for study based on response to systemic \\
& chemotherapy; role of patient choice/refusal unclear \\
Uygur et al. [22] (MA) & Refused RC \\
Villavicencio et al. [23] (MA) & - \\
\hline
\end{tabular}

(MA) included in meta-analysis.

only, and three used carboplatin-based regimens only. The number of cycles of systemic chemotherapy used ranged from one to twelve, with three as the most frequently reported median number of cycles. As shown in Table 3, the reported technique and number of transurethral resections performed varied considerably between studies. While one study reported that all patients in the systemic chemotherapy + TURBT cohort underwent radical TURBT that involved intentional perforation into the perivesical fat, [17] most studies did not comment on the radicality of the transurethral resection. Most studies reported two or three transurethral resections or biopsies between staging, treatment, and re-staging, while two reported only one resection for staging.

Thirteen of the 18 studies reported some use of intravesical chemotherapy in included patients. In three of these studies, all patients received intravesical treatment - bacillus Calmette-Guérin (BCG) in two and epirubicin or pirarubicin in one - as part of the study's primary treatment. Eight studies reported use of intravesical BCG or Mitomycin C only in patients with recurrent noninvasive disease. Of the remaining studies, two reported intravesical chemotherapy use prior to the beginning of the study and five did not report any use of intravesical therapy.

Patients included from six studies comprised only complete responders (cT0) at post-systemic chemotherapy re-staging, those from two included complete and partial responders, and patients from five studies included complete, partial, and nonresponders. The remaining five studies either did not re-stage or did not report post-systemic chemotherapy clinical staging. Only complete responders (cT0) were included in the meta-analysis.

As indicated in Table 2, the majority of studies included patients who refused RC, though five studies reported including or being limited to patients who were deemed unfit for radical surgery. Patient functional performance status and degree of comorbidity were not uniformly reported across studies. Six studies reported ECOG/Zubrod/WHO performance scores, all of which were $\leq 2$, and four reported Karnofsky performance scores with medians ranging $80-100 \%$ (overall range $40-100 \%$ ). These scores reflect that, per median values, patients in these studies were ambulatory and capable of self-care at the time of treatment $[28,29]$.

Two studies reported measures of comorbidity. The study by Bamias et al., [26] which was excluded from our meta-analysis, reported a median Charlson comorbidity score of 5 , which is associated with a significantly elevated mortality rate in the perioperative setting, for their entire study group [30]. Among this group, patients receiving systemic chemotherapy + TURBT were only those deemed unfit for radical cystectomy. The study by Lodde et al. [31] reported comorbidity in terms of ASA (American Society of Anesthesiologists) score, with a median score of 3, reflecting "severe systemic disease" [32]. Patients in this study reportedly entered the systemic 
Table 3

Treatment methods

\begin{tabular}{|c|c|c|c|c|c|}
\hline Authors & $\begin{array}{l}\text { Type of Systemic } \\
\text { Chemotherapy Given }\end{array}$ & $\begin{array}{l}\text { \# Cycles of Systemic } \\
\text { Chemotherapy in } \\
\text { Initial Treatment }\end{array}$ & $\begin{array}{l}\text { Intravesical Chemotherapy } \\
\text { Use }\end{array}$ & $\begin{array}{l}\text { Type of Intravesical } \\
\text { Chemotherapy } \\
\text { Given }\end{array}$ & $\begin{array}{l}\text { Description of Transurethral Resections } \\
\text { and Biopsies* }\end{array}$ \\
\hline Bamias et al. [26] & $\begin{array}{l}\text { Carboplatin + Gemcitabine or } \\
\text { Methotrexate + Carboplatin } \\
\text { + Vinblastine }\end{array}$ & Median $7(2-12)$ & Not used/use not addressed & $\mathrm{n} / \mathrm{a}$ & 1. Staging TURBT \\
\hline Damyanov et al. [27] & $\mathrm{CMV}$ & 3 & $\begin{array}{l}\text { Received by all patients as } \\
\text { part of initial treatment }\end{array}$ & BCG & $\begin{array}{l}\text { 1. Staging: not specified } \\
\text { 2. Pre-systemic chemotherapy } \\
\text { cystoscopy with biopsy } \\
\text { 3. Post-systemic chemotherapy restaging } \\
\text { TURBT for partial responders; biopsy } \\
\text { for others }\end{array}$ \\
\hline de la Rosa et al. [15] (MA) & CMV & 6 & $\begin{array}{l}\text { For recurrent non-invasive } \\
\text { disease only }\end{array}$ & BCG & $\begin{array}{l}\text { 1. Staging TURBT } \\
\text { 2. Pre-systemic chemotherapy TURBT } \\
\text { 3. Post-systemic chemotherapy } \\
\text { restaging: not specified }\end{array}$ \\
\hline deVere White et al. [34] (MA) & $\begin{array}{l}\text { Paclitaxel + Carboplatin + } \\
\text { Gemcitabine }\end{array}$ & 3 & Used previously only & - & $\begin{array}{l}\text { 1. Staging TURBT } \\
\text { 2. Pre-systemic chemotherapy TURBT } \\
\text { 3. Post-systemic chemotherapy restaging } \\
\text { TURBT }\end{array}$ \\
\hline Given et al. [33] (MA) & CMV +/- Doxorubicin & 2,3 & $\begin{array}{l}\text { For recurrent non-invasive } \\
\text { disease only }\end{array}$ & BCG or Mitomycin C & $\begin{array}{l}\text { 1. Staging: not specified } \\
\text { 2. Pre-systemic chemotherapy TURBT } \\
\text { 3. Post-systemic chemotherapy restaging } \\
\text { TURBT }\end{array}$ \\
\hline Herr [10] (MA) & Cisplatin-based (81\% MVAC) & $4+$ & $\begin{array}{l}\text { For recurrent non-invasive } \\
\text { disease only }\end{array}$ & BCG & $\begin{array}{l}\text { 1. Staging TURBT } \\
\text { 2. Pre-systemic chemotherapy TURBT } \\
\text { 3. Post-systemic chemotherapy restaging } \\
\text { biopsy }\end{array}$ \\
\hline Herr et al. [16] (MA) & MVAC & 4 & $\begin{array}{l}\text { For recurrent non-invasive } \\
\text { disease only }\end{array}$ & BCG & $\begin{array}{l}\text { 1. Staging: not specified } \\
\text { 2. Pre-systemic chemotherapy biopsy } \\
\text { 3. Post-systemic chemotherapy restaging } \\
\text { TURBT }\end{array}$ \\
\hline Kondás et al. [17] & $\begin{array}{l}\text { Cisplatin }+5-\mathrm{FU}+ \\
\text { [Adriamycin or } \\
\text { Mitomycin] }\end{array}$ & $1(n=7), 2(n=26)$ & Not used/use not addressed & $\mathrm{n} / \mathrm{a}$ & $\begin{array}{l}\text { 1. Staging biopsy } \\
\text { 2. Pre-systemic chemotherapy TURBT }\end{array}$ \\
\hline Kuroiwa et al. [18] (MA) & MVAC +/- Angiotensin II & Median $3(2-4)$ & Not used/use not addressed & $\mathrm{n} / \mathrm{a}$ & $\begin{array}{l}\text { 1. Staging biopsy } \\
\text { 2. Post-systemic chemotherapy restaging } \\
\text { TURBT }\end{array}$ \\
\hline Lekili et al. [19] & MVAC & Median 3 & Not used/use not addressed & $\mathrm{n} / \mathrm{a}$ & $\begin{array}{l}\text { 1. Staging TURBT } \\
\text { 2. Post-systemic chemotherapy restaging } \\
\text { TURBT/biopsy }\end{array}$ \\
\hline
\end{tabular}


Table 3

\begin{tabular}{|c|c|c|c|c|c|}
\hline Authors & $\begin{array}{l}\text { Type of Systemic } \\
\text { Chemotherapy Given }\end{array}$ & $\begin{array}{l}\text { \# Cycles of Systemic } \\
\text { Chemotherapy in } \\
\text { Initial Treatment }\end{array}$ & $\begin{array}{l}\text { Intravesical Chemotherapy } \\
\text { Use }\end{array}$ & $\begin{array}{l}\text { Type of Intravesical } \\
\text { Chemotherapy } \\
\text { Given }\end{array}$ & $\begin{array}{l}\text { Description of Transurethral Resections } \\
\text { and Biopsies* }\end{array}$ \\
\hline Li et al. [20] & Gemcitabine + Cisplatin & 8 & $\begin{array}{l}\text { Received by all patients as } \\
\text { part of initial treatment }\end{array}$ & $\begin{array}{l}\text { Epirubicin or } \\
\text { Pirarubicin }\end{array}$ & $\begin{array}{l}\text { 1. Staging biopsy } \\
\text { 2. Pre-systemic chemotherapy TURBT } \\
\text { 3. +/- Re-TURBT within 4-6 weeks, } \\
\text { depending on pathology of prior } \\
\text { TURBT } \\
\text { 4. Post-systemic chemotherapy restaging } \\
\text { biopsy }\end{array}$ \\
\hline Lodde et al. [31] & $\mathrm{CMV}$ & 2 & Not used/use not addressed & $\mathrm{n} / \mathrm{a}$ & $\begin{array}{l}\text { 1. Staging TURBT } \\
\text { 2. Pre-systemic chemotherapy TURBT }\end{array}$ \\
\hline Matulay et al. [24] (MA) & $\begin{array}{l}\text { Platinum-based (specific } \\
\text { regimen up to physician } \\
\text { discretion): MVAC, } \\
\text { Gemcitabine + Cisplatin, or } \\
\text { other/unknown recurrences }\end{array}$ & - & $\begin{array}{l}\text { For recurrent non-invasive } \\
\text { disease only }\end{array}$ & BCG & $\begin{array}{l}\text { 1. Staging TURBT } \\
\text { 2. Post-systemic chemotherapy restaging } \\
\text { biopsy }\end{array}$ \\
\hline Shimizu et al. [21] & $\begin{array}{l}\text { Cisplatin }+ \text { Methotrexate }+ \\
\text { Doxorubicin }\end{array}$ & 2 & $\begin{array}{l}\text { Received by all patients as } \\
\text { part of initial treatment }\end{array}$ & BCG & 1. Staging TURBT \\
\hline Solsona et al. [9] & $\begin{array}{l}\text { CMV }(n=14), \text { MVAC } \\
\quad(n=39), \text { Gemcitabine }+ \\
\text { Cisplatin }(n=19) \\
\text { Gemcitabine + Carboplatin } \\
(n=3)\end{array}$ & 3 & $\begin{array}{l}\text { For recurrent non-invasive } \\
\text { disease only }\end{array}$ & BCG or Mitomycin C & $\begin{array}{l}\text { 1. Staging TURBT } \\
\text { 2. Pre-systemic chemotherapy biopsy } \\
\text { 3. Post-systemic chemotherapy: not } \\
\text { specified }\end{array}$ \\
\hline Sternberg et al. [11] (MA) & MVAC & 3 & $\begin{array}{l}\text { For recurrent non-invasive } \\
\text { disease only }\end{array}$ & BCG & $\begin{array}{l}\text { 1. Staging TURBT } \\
\text { 2. Post-systemic chemotherapy restaging } \\
\text { TURBT } \\
\text { 3. Additional TURBT }\end{array}$ \\
\hline Uygur et al. [22] (MA) & MVEC & 4 & Used previously only & BCG & $\begin{array}{l}\text { 1. Pre-systemic chemotherapy TURBT } \\
\text { 2. Post-systemic chemotherapy restaging } \\
\text { TURBT }\end{array}$ \\
\hline Villavicencio et al. [23] (MA) & $\begin{array}{l}\text { Carboplatin }+ \text { Vinblastine }+ \\
\text { Methotrexate }\end{array}$ & 3 & $\begin{array}{l}\text { For recurrent non-invasive } \\
\text { disease only }\end{array}$ & BCG & $\begin{array}{l}\text { 1. Staging TURBT } \\
\text { 2. Pre-systemic chemotherapy TURBT } \\
\text { 3. Post-systemic chemotherapy restaging } \\
\text { TURBT }\end{array}$ \\
\hline
\end{tabular}

(MA) included in meta-analysis. * refers to staging and treatment in the period immediately surrounding systemic chemotherapy; does not include additional biopsies and resections for post-treatment surveillance and/or treatment of recurrence. 
Table 4

Follow-up and survival outcomes

\begin{tabular}{|c|c|c|c|c|c|c|c|}
\hline Authors & $\begin{array}{l}\text { Median F-U (range) } \\
{[\text { mo.] }}\end{array}$ & Follow-up Strategy Described & $\mathrm{OS}$ at F-U & DSS at F-U & 5-year OS & 5-year DSS & $\begin{array}{l}\text { Other Reported } \\
\text { Survival Outcome }\end{array}$ \\
\hline Bamias et al. & $68^{*}$ & - & - & - & $20 \%$ & $23 \%$ & - \\
\hline Damyanov et al. & $21.6(7-48)$ & $\begin{array}{l}\text { Cystoscopy with biopsy, cytology, clinical exam, } \\
\text { CT +/- ultrasound (q3 months for } 2 \text { years, q6 } \\
\text { months for 3rd year, q1 year after that) }\end{array}$ & - & $85.7 \%$ & - & - & - \\
\hline de la Rosa et al. (MA) & $78(70-109)$ & 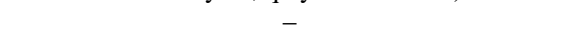 & $66.7 \%$ & $72.2 \%$ & - & - & - \\
\hline deVere White et al. (MA) & $22 *$ & - & $76 \%$ & - & - & - & - \\
\hline Given et al. (MA) & 60 & $\begin{array}{l}\text { Cystoscopy }+/ \text { - biopsy (q3 months for } 2 \text { years, } \\
\text { then q6 months if no recurrence); IVU and CXR } \\
\text { (q1 year); other studies "as clinically indicated" }\end{array}$ & $43 \%$ & $43 \%$ & - & - & - \\
\hline Herr, 2008 (MA) & 86 & Cystoscopy +/- TUR, CTAP (q3-6 months) & $64 \%$ & $64 \%$ & - & - & - \\
\hline Herr et al., 1998 (MA) & $120(96-156)$ & Cystoscopy and TUR ("as needed" q3-6 months) & $75 \%$ & - & - & - & - \\
\hline Kondás et al. & mean: $34(4-71)$ & $\begin{array}{l}\text { Cystoscopy +/- biopsy, cytology, ultrasound (q3 } \\
\text { months); CT, urography, bone scan, CXR, (after } \\
6 \text { months, then q1 year) }\end{array}$ & - & - & $26.5 \%$ & $53.3 \%$ & - \\
\hline Kuroiwa et al. (MA) & $23.5(14-31+)$ & 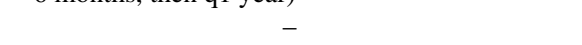 & $83.3 \%$ & $100 \%$ & - & - & - \\
\hline Lekili et al. & $33.6^{*}$ & $\begin{array}{l}\text { Cystoscopy with biopsy, CT } \\
\text { abdomen/pelvis/thorax, visual and physical } \\
\text { exam, bimanual exam }\end{array}$ & $83.3 \%$ & $83.3 \%$ & - & - & - \\
\hline Li et al. & mean: $58.4(26-102)$ & $\begin{array}{l}\text { "overall follow-up... including lung checking, } \\
\text { bone scans, and liver examinations" (q3 months) }\end{array}$ & - & - & - & - & $\begin{array}{l}\text { 88.9\% 2-year OS; } \\
94.4 \% \text { 2-year DSS }\end{array}$ \\
\hline Lodde et al. & $26.9(24.6-34.3)$ & $\begin{array}{l}\text { Cystoscopy and cytology (q3 months); CTAP and } \\
\text { CXR (q6 months); IV pyelogram (q1 year) }\end{array}$ & $25 \%$ & $25 \%$ & - & - & - \\
\hline Matulay et al. (MA) & $37.4^{*}$ & Cystoscopy with biopsy, cytology, CTAP & $77.8 \%$ & $91.7 \%$ & - & - & - \\
\hline Shimizu et al. & $73(8-139)^{*}$ & $\begin{array}{l}\text { Cystoscopy and cytology (q3 months for } 3 \text { years, } \\
\text { q6 months for } 3-5 \text { years, q1 year after } 5 \text { years); } \\
\text { IV pyelography, pelvic CT, and CXR ( } 66 \\
\text { months for } 3 \text { years, then q1 year) }\end{array}$ & - & - & $87.5 \%$ & $87.5 \%$ & - \\
\hline Solsona et al. & $55(9-211)$ & $\begin{array}{l}\text { Cystoscopy and bimanual EUA, random bladder } \\
\text { mucosa biopsies, TUR at original tumor site, } \\
\text { and cytology (q3 months for } 2 \text { years, q4 months } \\
\text { for next } 2 \text { years, q6 months until year 5, then q1 } \\
\text { year); CTAP or MRI, CXR (q3 months for } 1 \\
\text { year, then q6 months for } 4 \text { years) }\end{array}$ & - & - & - & $64.5 \%$ & - \\
\hline Sternberg et al. (MA) & $56+(10-160+)$ & Cystoscopy and cytology (q3 months) & $60 \%$ & - & $67 \%$ & - & - \\
\hline Uygur et al. (MA) & mean: $41(8-58+)$ & $\begin{array}{l}\text { Cystoscopy with deep biopsy (q3 months); IVU + } \\
\text { CT (q1 year) }\end{array}$ & $75 \%$ & $81.2 \%$ & - & - & - \\
\hline Villavicencio et al. (MA) & $98.5(13-246)^{*}$ & $\begin{array}{l}\text { Cystoscopy, cytology (q3 months for first } 3 \text { years, } \\
\text { q6 mo. for next } 2 \text { years, then q1 year); CXR and } \\
\text { CTAP (q1 year) }\end{array}$ & $62.8 \%$ & $81.4 \%$ & - & - & - \\
\hline
\end{tabular}

MA) included in meta-analysis. *value applies to entire study group, not just those meeting clinical criteria for inclusion in this analysis. F-U: Follow-up. IVU: intravenous urography. CXR: chest x-ray. CTAP: CT abdomen and pelvis. EUA: exam under anesthesia. 
a

\begin{tabular}{|c|c|c|}
\hline \multirow{4}{*}{$\begin{array}{l}\text { de la Rosa et al. } \\
\text { deVere White et al. } \\
\text { Given et al. } \\
\text { Herr, } 2008\end{array}$} & $\mapsto-1$ & $0.01[0.00,0.01]$ \\
\hline & & $0.01[0.00,0.02]$ \\
\hline & & $-0.01[0.00,0.03]$ \\
\hline & $1=-1$ & $0.01[0.00,0.01]$ \\
\hline Herr et al., 1998 & $\square$ & $0.00[0.00,0.00]$ \\
\hline Matulay et al. & $\longmapsto$ & $0.01[0.00,0.01]$ \\
\hline Sternberg et al. & $\mapsto-1$ & $0.01[0.00,0.01]$ \\
\hline Uygur et al. & 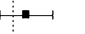 & $0.00[-0.00,0.01]$ \\
\hline \multirow[t]{2}{*}{ Villavicencio et al. } & $r$ 마 & $0.00[0.00,0.01]$ \\
\hline & $\bullet$ & $0.01[0.00,0.01]$ \\
\hline-0.01 & $\begin{array}{r}0.0 \\
\text { azard }\end{array}$ & 0.03 \\
\hline
\end{tabular}

b

Survival Rates and Meta-Analysis Result (95\% Cl)

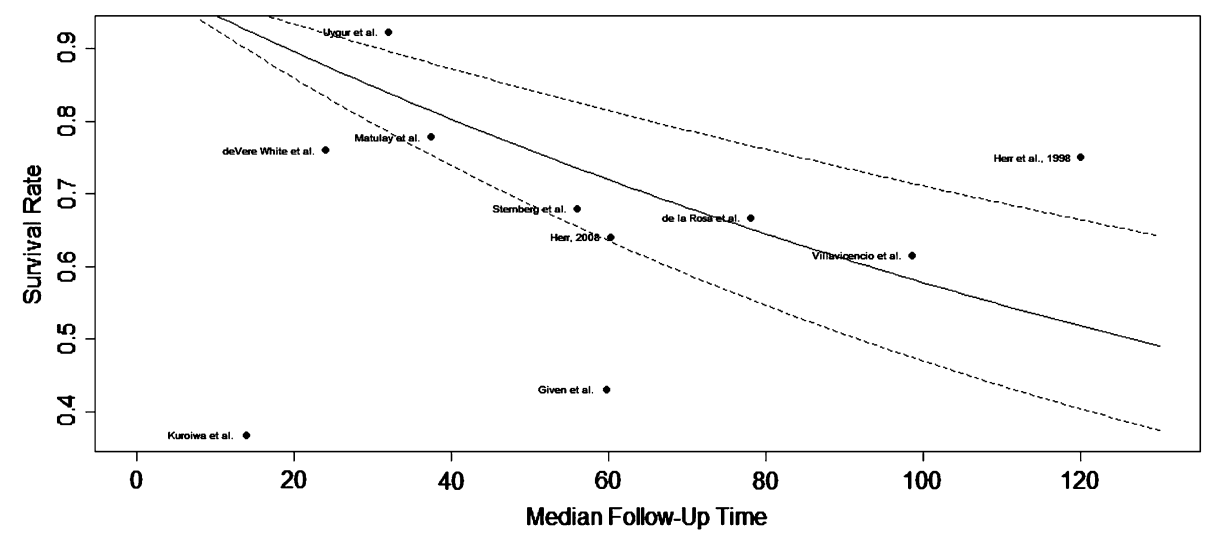

Fig. 2. (a) Forest plot depicting hazard rates, signifying rate of death, in studies included in the meta-analysis. (b) Estimated Kaplan-Meier curve overlay on the survival rates from each study included in the meta-analysis.

chemotherapy + TURBT cohort due to "Advanced age, patients' choice, and/or due to co-morbid disease with high ASA score." While no other studies reported a numeric assessment of comorbidity, those by Given et al., [33] Kondás et al., [17] Kuroiwa et al., [18] and $\mathrm{Li}$ et al. [20] all indicated that at least part of their bladder sparing cohorts had been selected due to poor physical status, underlying disease, or unspecified surgical risk or inoperability.

\section{Results of individual studies}

Table 4 summarizes the survival data extracted for each included study, including those with patients who did not achieve a complete clinical response to systemic chemotherapy as well as those that only included complete responders. OS ranged from $20 \%$ to $87.5 \%$ at times ranging from four months to ten years post-treatment. Four studies reported 5-year OS for all included patients and one provided 2-year OS for all patients. The eleven other studies that reported or permitted extrapolation of OS provided only the survival at each patient's time of latest followup. Two studies reported DSS as the only survival data.

\section{Meta-analysis}

Hazard rate, i.e. mortality rate, estimates and their standard deviations were obtained for patients from 10 out of the 18 studies under the exponential survival rate assumption. The $p$-value of the test of heterogeneity is 0.054 , so a random effects model was used to conduct meta-analysis for mortality rate. The $\mathrm{I}^{2}$ is $57.8 \%$. The forest plot for nine of ten studies is shown in Fig. 2a. One study [18] contributed just one patient to the meta-analysis and therefore showed much larger variance. This study was withheld from the forest plot. Fig. 2b shows the estimated KaplanMeier curve overlay on the individual survival rates. The estimated 5-year survival rate is $72 \%$ with a $95 \%$ confidence interval [64\%, $82 \%]$. 


\section{DISCUSSION}

The significant potential (30-40\%) for complete pathological remission of MIBC at the time of RC in patients treated with complete TURBT and NAC compels investigation into the safety of bladder-sparing approaches to the disease. Through systematic review of the literature, we were able to identify 10 studies that included clinical complete responders following only TURBT and systemic chemotherapy with sufficient quality and reporting for meta-analysis. The estimated 5-year survival rate for all patients included from these studies is $72 \%$, with statistically significant heterogeneity between studies. Attempts to compare systemic chemotherapy regimens (carboplatin-based vs. cisplatin-based) were limited by a lack of power deriving from unbalanced subgroups. Of the four studies that used carboplatin-based regimens, one [26] included only patients who were deemed "unfit for radical cystectomy" and reported $20 \%$ overall survival at 5 years, [26] while the others either included patients who refused RC [9, 34] or did not specify the reason for bladder sparing, [23] and reported rates closer to the $72 \%$ 5-year survival estimated by this meta-analysis.

The concept of treating MIBC with a highly conservative approach is not new, given that the advanced age of many bladder cancer patients makes them unfit for RC and the associated morbidity. Transurethral resection of bladder tumor alone was reported by Herr in a 2001 study with 10-year survival outcomes [35]. In this cohort, radical TURBT alone was associated with a DSS of $76 \%$ for 99 patients with T0 or T1 post-TURBT, by comparison with $71 \%$ in the 52 post-TURBT T0 or T1 patients who had immediate cystectomy. Similarly, Solsona et al. reported 5- and 10 -year DSS of $81.9 \%$ and $79.5 \%$ in 133 patients undergoing definitive treatment by radical TUR with negative biopsies of the tumor bed [36].

The efficacy of NAC for eliminating pathologically detectable bladder cancer and improving overall survival in MIBC is now well demonstrated. One landmark randomized phase III trial, SWOG 8710 , showed a significant improvement in OS in favor of neoadjuvant MVAC preceding RC versus RC alone (mean 77 months vs. 46 months) for patients with locally advanced urothelial cancer [7] Importantly, the study showed a survival advantage for all patients who had achieved complete pathological remission by the time of cystectomy (pT0), whether following combination MVAC and TURBT or TURBT alone. Notably, a significantly higher proportion of the MVAC arm achieved pT0 (38\% vs. $15 \%$ ), raising the possibility that a significant portion of MIBC patients have been cured prior to radical cystectomy. While it is not uncommon for patients to refuse cystectomy following TURBT and systemic chemotherapy, $[9,10,16,18,22,24,27,33]$ especially if there is no evidence of disease on re-staging, the outcomes of these patients are not well documented. This is largely because cystectomy outcomes are usually not reported on an intention to treat basis, meaning that patients who ultimately refuse RC or are found to be inappropriate surgical candidates are typically excluded [11].

Some groups have published studies with the specific aim of reporting the outcomes of an MIBC cohort who receives TURBT and systemic chemotherapy together as definitive treatment, typically in a non-randomized comparison with a contemporary or historical cohort receiving RC following NAC and TURBT [9, 10, 15-17, 19-23, $34,37]$. Others present data on the definitive systemic chemotherapy plus TURBT cohort as one subset of a larger bladder sparing group that includes patients who received partial cystectomy, [11] radiotherapy, $[26,31,33]$ or systemic chemotherapy alone $[18,27]$. These studies are typically smaller, often case series from single institutions. Even the multi-institutional SWOG 0219 trial [34] required 5 years to accrue their 77 patients, despite the benefit of NAC reported in the aforementioned SWOG 8710 trial [7]. Therefore, the clinical impact of these reports is limited, indicating a need for a systematic review and meta-analysis of these data together.

A significant limitation in the generalizability of the data included in our analysis is the variability in TURBT technique across physicians and practices. Studies included in our analysis varied in the number of TURBTs patients had, and mostly did not address the radicality of these resections. The 2017 guidelines for MIBC issued by the American Urologic Association (AUA) recommend "maximal transurethral resection to remove all visible disease" when pursuing bladder preservation, a recommendation that the group derived from multiple prospective studies of TMT showing approximately $20 \%$ higher rates of local control following a visibly complete TURBT [38-40]. This center's practice and recommendation for maximal TURBT includes, when safe, resection deep into the perivesical fat including a wide margin of resection with no gross tumor left behind, with staging by pathologic analysis as 
well as cross sectional imaging using CT or MRI. In a 2008 prospective analysis of patients refusing cystectomy after receiving NAC, Herr found that all patients referred for cystectomy following initial transurethral resection by the referring urologist had residual muscle invasion when re-staged by TUR [10]. Furthermore, patients who achieved complete resection on repeat TURBT (i.e. no microscopic evidence of tumor in contiguous muscle tissue deep and lateral to the primary tumor site) had a 93\% survival, versus $38 \%$ in those with incomplete resection on repeat TURBT, defined as having tumor present in biopsies of deep muscle or fat adjacent to the primary mass. These findings underscore the importance of high-quality TURBT in treating MIBC, specifically in those undergoing definitive treatment with systemic chemotherapy or who desire bladder-sparing approaches.

Radical cystectomy series highlight the favorable prognostic significance of a complete response to NAC for survival. A 2001 phase III trial reported a close correlation between preoperative downstaging with systemic chemotherapy and cure rate, with $88 \%$ of pT0 patients having no recurrent disease at nearly 7 years median follow-up compared with $86 \%$ relapse and death in the group found with node positive disease at cystectomy [41]. As these authors comment, it is impossible to know whether this advantage is a result of the ability of systemic chemotherapy to make MIBC more susceptible to cure by TURBT, or rather evidence of systemic chemotherapy selecting out the patients with the most favorable biology.

Whether it bespeaks a true clinical benefit of cT0 status in bladder sparing or simply suggests a screening method for bladder cancers more amenable to these regimens, the evidence for improved outcomes when following clinical complete remission with systemic chemotherapy indicates the importance of careful patient selection when considering bladdersparing treatment. In light of this, the effectiveness of TUR as a staging method is of great interest. The aforementioned phase II SWOG 0219 trial - in which patients were offered bladder sparing if they achieved cT0 on re-staging TUR following combination TURBT and a systemic chemotherapy regimen of paclitaxel, carboplatin, and gemcitabine - importantly found that, of the 10 patients who achieved cT0 after TURBT and chemotherapy but elected immediate cystectomy, 6 were found with residual invasive disease upon cystectomy [34]. These results challenge the adequacy of TURBT to detect all microscopic disease in deep muscle and perivesical fat, which may also be variable depending upon the physician.

Given the imperfect ability to predict recurrence and progression following bladder-sparing treatment, the safety of a recommendation in favor of bladder preservation relies upon salvage cystectomy being an effective means of curing recurrent invasive cancer. The frequency of salvage cystectomy was not analyzed in this meta-analysis due to limited reporting, though the mean among studies included in the metaanalysis that did report it was approximately $33 \%$ (range $0 \%$ to $65 \%$ ). Salvage cystectomy outcomes are predictably poor as these patients have aggressive disease that has already failed bladder-sparing treatment. However, the 2008 study by Herr reported that nearly half of the $22 \%$ of patients who required cystectomy for recurrent or new MIBC after attempted bladder sparing were successfully salvaged [10]. In a 2009 editorial [42]. Herr estimates that refusing cystectomy after a complete response carries an added mortality risk between $7 \%$ and $16 \%$, as some patients will recur with invasive disease and cannot be salvaged. As Herr comments, this additional risk of death may be acceptable to some patients motivated to preserve their bladders, while it may be unacceptably high for others.

Our analysis has its limits, both at the level of the studies included and at the review level. There is unquantifiable variability in the practice and intensity of TURBT between physicians, both at the level of staging and treatment. There is notable heterogeneity among the included studies in this respect, with some performing only a single staging TURBT and others performing multiple resections. Additionally, staging was dependent upon pathological assessment, which may introduce interobserver variability.

The studies included in this analysis are susceptible to selection bias. The nature of this bias is not uniform, however, as some studies include primarily younger, healthier patients opting for less treatment for the sake of preserving of their bladders and sexual function, while other studies included groups of patients foregoing RC due to advanced age and/or comorbid disease. In addition to the selection process, bias may manifest in how carefully patients are followed after treatment. However, patients who have opted for bladder sparing are intentionally a highly selected group, and some element of patient selection bias is required for this treatment to be effective.

At the review level, this study is limited by our inability to include all patients who met the appropriate clinical criteria and underwent definitive systemic 
chemotherapy and TURBT. As shown in Fig. 1, 31 additional publications were found in the search process but were not included because survival was not reported and could not be extrapolated for the distinct cohort of interest. For example, if a particular publication's bladder sparing outcome data included that of patients who had received systemic chemotherapy plus partial cystectomy along with the systemic chemotherapy plus TURBT cohort, none of the definitive TURBT plus systemic chemotherapy group in this cohort could be counted. Furthermore, variable reporting within studies limited our ability to compare other outcomes such as recurrence, PFS, cystectomy-free survival, and DSS.

Finally, our survival analysis is limited by the heterogeneity of the data reported between studies. Given that patient selection for bladder sparing is clinically important, it is noteworthy that our analysis included patients who were chosen for bladder sparing for different reasons, with some having elected this option and others having been deemed unfit for surgery. Studies also varied in follow-up time, follow-up modalities, and, we may infer, quality of follow-up. This is especially important in bladder sparing, where a delayed cystectomy may salvage survival in patients with recurrent disease.

\section{CONCLUSIONS}

This meta-analysis finds an estimated 5-year OS of $72.0 \%$ for patients with locally invasive bladder cancer achieving complete clinical response following definitive treatment with TURBT plus systemic chemotherapy. Further study is needed to determine how to improve patient selection for bladder-sparing therapy, including identifying novel biomarkers that can predict a complete response to chemotherapy.

\section{CONFLICT OF INTEREST}

The authors have no conflict of interest to report.

\section{SUPPLEMENTARY MATERIAL}

The supplementary material is available in the electronic version of this article: http://dx.doi.org/ 10.3233/BLC-170134.

\section{REFERENCES}

[1] Siegel RL, Miller KD, Jemal A. Cancer Statistics, 2017. CA Cancer J Clin 2017;67(1):7-30.
[2] Burger M, Catto JW, Dalbagni G, Grossman HB, Herr $\mathrm{H}$, Karakiewicz P, et al. Epidemiology and risk factors of urothelial bladder cancer. Eur Urol 2013;63(2):234-41.

[3] Chang SS, Bochner BH, Chou R, Dreicer R, Kamat AM, Lerner SP, et al. Treatment of non-metastatic muscleinvasive bladder cancer: AUA/ASCO/ASTRO/SUO Guideline. J Urol 2017.

[4] Alfred Witjes J, Lebret T, Comperat EM, Cowan NC, De Santis M, Bruins HM, et al. Updated 2016 EAU guidelines on muscle-invasive and metastatic bladder cancer. Eur Urol 2017;71(3):462-75.

[5] International Collaboration of T, Medical Research Council Advanced Bladder Cancer Working P, European Organisation for R, Treatment of Cancer Genito-Urinary Tract Cancer G, Australian Bladder Cancer Study G, National Cancer Institute of Canada Clinical Trials G, et al. International phase III trial assessing neoadjuvant cisplatin, methotrexate, and vinblastine chemotherapy for muscleinvasive bladder cancer: Long-term results of the BA06 30894 trial. J Clin Oncol 2011;29(16):2171-7.

[6] Bochner BH, Dalbagni G, Sjoberg DD, Silberstein J, Keren Paz GE, Donat SM, et al. Comparing open radical cystectomy and robot-assisted laparoscopic radical cystectomy: A randomized clinical trial. Eur Urol 2015;67(6): 1042-50.

[7] Grossman HB, Natale RB, Tangen CM, Speights VO, Vogelzang NJ, Trump DL, et al. Neoadjuvant chemotherapy plus cystectomy compared with cystectomy alone for locally advanced bladder cancer. N Engl J Med 2003;349(9): 859-66.

[8] Meyer A, Ghandour R, Bergman A, Castaneda C, Wosnitzer M, Hruby G, et al. The natural history of clinically complete responders to neoadjuvant chemotherapy for urothelial carcinoma of the bladder. J Urol 2014;192(3):696-701.

[9] Solsona E, Climent MA, Iborra I, Collado A, Rubio J, Ricos $\mathrm{JV}$, et al. Bladder preservation in selected patients with muscle-invasive bladder cancer by complete transurethral resection of the bladder plus systemic chemotherapy: Long-term follow-up of a phase 2 nonrandomized comparative trial with radical cystectomy. Eur Urol 2009;55(4): 911-9.

[10] Herr HW. Outcome of patients who refuse cystectomy after receiving neoadjuvant chemotherapy for muscle-invasive bladder cancer. Eur Urol 2008;54(1):126-32.

[11] Sternberg CN, Pansadoro V, Calabro F, Schnetzer S, Giannarelli D, Emiliozzi P, et al. Can patient selection for bladder preservation be based on response to chemotherapy? Cancer 2003;97(7):1644-52.

[12] Giacalone NJ, Shipley WU, Clayman RH, Niemierko A, Drumm M, Heney NM, et al. Long-term outcomes after bladder-preserving tri-modality therapy for patients with muscle-invasive bladder cancer: An updated analysis of the massachusetts general hospital experience. Eur Urol 2017;71(6):952-60.

[13] James ND, Hussain SA, Hall E, Jenkins P, Tremlett J, Rawlings C, et al. Radiotherapy with or without chemotherapy in muscle-invasive bladder cancer. $\mathrm{N}$ Engl $\mathrm{J}$ Med 2012;366(16):1477-88.

[14] Krause FS, Walter B, Ott OJ, Haberle L, Weiss C, Rodel C, et al. 15-year survival rates after transurethral resection and radiochemotherapy or radiation in bladder cancer treatment. Anticancer Res 2011;31(3):985-90.

[15] de la Rosa F, Garcia-Carbonero R, Passas J, Rosino A, Lianes P, Paz-Ares L. Primary cisplatin, methotrexate and vinblastine chemotherapy with selective bladder 
preservation for muscle invasive carcinoma of the bladder: Long-term followup of a prospective study. J Urol 2002;167(6):2413-8.

[16] Herr HW, Bajorin DF, Scher HI. Neoadjuvant chemotherapy and bladder-sparing surgery for invasive bladder cancer: Ten-year outcome. J Clin Oncol 1998;16(4):1298-301.

[17] Kondás J, Engloner L, Vaczi L, Konder G. Transurethral resection and intra-arterial chemotherapy for muscleinvasive bladder cancer. Int Urol Nephrol 1996;28(2):181-7.

[18] Kuroiwa T, Naito S, Hasuo K, Kishikawa T, Masuda K, Kumazawa J. Phase II study of a new combined primary chemotherapy regimen, intravenous methotrexate and vincristine and intraarterial adriamycin and cisplatin, for locally advanced urinary bladder cancer: Preliminary results. Cancer Chemother Pharmacol 1995;35(5):357-63.

[19] Lekili M, Ayder AR, Minareci S, Nergis S, Durgun MN. Conservative management of advanced bladder cancer. Int Urol Nephrol 1995;27(6):747-52.

[20] Li J, Wang Q, Xiao B, Zhang X. Effect of internal iliac artery chemotherapy after transurethral resection of bladder tumor for muscle invasive bladder cancer. Chin J Cancer Res 2014;26(5):558-63.

[21] Shimizu H, Nishimura T, Kimura G, Matsuzawa I, Kondo Y. Effect of adjuvant transarterial infusion of anticancer agents after transurethral resection in patients with pT2a muscle invasive transitional cell carcinoma of the bladder: Five and ten-year outcome. J Nippon Med Sch 2004;71(4):263-9.

[22] Uygur MC, Yaman I, Altug U, Erol D. Conservative management of stage T2 or T3a bladder cancer with deep transurethral resection followed by four cycles of chemotherapy. Br J Urol 1996;78(2):201-4.

[23] Villavicencio H, Rodriguez Faba O, Palou J, Gausa L, Algaba F, Marcuello E. Bladder preservation strategy based on combined therapy in patients with muscle-invasive bladder cancer: Management and results at long-term follow-up. Urol Int 2010;85(3):281-6.

[24] Matulay JT VM, Onyeji I, Meyer A, RoyChoudhury A, Benson MC, Wenske S, Anderson CB, McKiernan JM, DeCastro GJ. Natural history of clinical complete response to neoadjuvant chemotherapy for urothelial carcinoma of the bladder: Updated single-institution experience. J Clin Oncol 2016;34(suppl):abstr 4537.

[25] Moher D, Liberati A, Tetzlaff J, Altman DG, Group P. Preferred reporting items for systematic reviews and meta-analyses: The PRISMA statement. PLoS Med 2009;6(7):e1000097.

[26] Bamias A, Tsantoulis P, Zilli T, Papatsoris A, Caparrotti F, Kyratsas $C$, et al. Outcome of patients with nonmetastatic muscle-invasive bladder cancer not undergoing cystectomy after treatment with noncisplatin-based chemotherapy and/or radiotherapy: A retrospective analysis. Cancer Med 2016;5(6):1098-107.

[27] Damyanov C, Tsingilev B, Tabakov V, Simeonov R. Organ-sparing treatment of invasive transitional cell bladder carcinoma. J BUON 2002;7(3):241-5.

[28] Crooks V, Waller S, Smith T, Hahn TJ. The use of the Karnofsky Performance Scale in determining outcomes and risk in geriatric outpatients. J Gerontol 1991;46(4): M139-44.

[29] Oken MM, Creech RH, Tormey DC, Horton J, Davis TE, McFadden ET, et al. Toxicity and response criteria of the Eastern Cooperative Oncology Group. Am J Clin Oncol 1982;5(6):649-55.
[30] Charlson M, Szatrowski TP, Peterson J, Gold J. Validation of a combined comorbidity index. J Clin Epidemiol 1994;47(11):1245-51.

[31] Lodde M, Palermo S, Comploj E, Signorello D, Mian C, Lusuardi L, et al. Four years experience in bladder preserving management for muscle invasive bladder cancer. Eur Urol 2005;47(6):773-8; discussion 8-9.

[32] Lupei MI, Chipman JG, Beilman GJ, Oancea SC, Konia MR. The association between ASA status and other risk stratification models on postoperative intensive care unit outcomes. Anesth Analg 2014;118(5):989-94.

[33] Given RW, Parsons JT, McCarley D, Wajsman Z. Bladdersparing multimodality treatment of muscle-invasive bladder cancer: A five-year follow-up. Urology 1995;46(4):499504; discussion -5 .

[34] deVere White RW, Lara PN Jr, Goldman B, Tangen CM, Smith DC, Wood DP Jr, et al. A sequential treatment approach to myoinvasive urothelial cancer: A phase II Southwest Oncology Group trial (S0219). J Urol 2009;181(6):2476-80; discussion 80-1.

[35] Herr HW. Transurethral resection of muscle-invasive bladder cancer: 10-year outcome. J Clin Oncol 2001;19(1): 89-93.

[36] Solsona E, Iborra I, Collado A, Rubio-Briones J, Casanova J, Calatrava A. Feasibility of radical transurethral resection as monotherapy for selected patients with muscle invasive bladder cancer. J Urol 2010;184(2):475-80.

[37] Liang S, Zou Q, Han B, Jing Y, Cui D, An X, et al. Intraarterial chemotherapy for muscle-invasive bladder cancer following transurethral resection. Urol Int 2015;94(4): 406-11

[38] Kaufman DS, Winter KA, Shipley WU, Heney NM, Chetner MP, Souhami L, et al. The initial results in muscleinvading bladder cancer of RTOG 95-06: Phase I/II trial of transurethral surgery plus radiation therapy with concurrent cisplatin and 5-fluorouracil followed by selective bladder preservation or cystectomy depending on the initial response. Oncologist 2000;5(6):471-6.

[39] Hagan MP, Winter KA, Kaufman DS, Wajsman Z, Zietman AL, Heney NM, et al. RTOG 97-06: Initial report of a phase I-II trial of selective bladder conservation using TURBT, twice-daily accelerated irradiation sensitized with cisplatin, and adjuvant MCV combination chemotherapy. Int J Radiat Oncol Biol Phys 2003;57(3):665-72.

[40] Kaufman DS, Winter KA, Shipley WU, Heney NM, Wallace HJ 3rd, Toonkel LM, et al. Phase I-II RTOG study (99-06) of patients with muscle-invasive bladder cancer undergoing transurethral surgery, paclitaxel, cisplatin, and twice-daily radiotherapy followed by selective bladder preservation or radical cystectomy and adjuvant chemotherapy. Urology 2009;73(4):833-7.

[41] Millikan R, Dinney C, Swanson D, Sweeney P, Ro JY, Smith TL, et al. Integrated therapy for locally advanced bladder cancer: Final report of a randomized trial of cystectomy plus adjuvant M-VAC versus cystectomy with both preoperative and postoperative M-VAC. J Clin Oncol 2001;19(20): 4005-13.

[42] Herr HW. Editorial comment on: Bladder preservation in selected patients with muscle-invasive bladder cancer by complete transurethral resection of the bladder plus systemic chemotherapy: Long-term follow-up of a phase 2 nonrandomized comparative trial with radical cystectomy. Eur Urol 2009;55(4):920-1. 